\title{
Recurrent Adult Immunoblastic Lymphoma
}

National Cancer Institute

\section{Source}

National Cancer Institute. Recurrent Adult Immunoblastic Lymphoma. NCI Thesaurus.

Code C8163.

The reemergence of adult immunoblastic lymphoma after a period of remission. 